\title{
Simultaneous bilateral pleomorphic adenomas of the parotid glands with unilateral tyrosine rich crystalloids
}

A T Warfield, L A Smallman

\begin{abstract}
A case of simultaneous, bilateral pleomorphic adenoma of the parotid glands following irradiation to the head and neck for childhood Hodgkin's disease is presented. The tumour on the side given the highest radiation exposure contained so-called tyrosine rich crystalloids (TRCs). Simultaneous salivary gland neoplasms are rare; TRCs are also an unusual finding. Simultaneous pleomorphic adenomas with TRCs confined to one tumour have never been described, and any association between TRCs and irradiation has never been commented on.

The precise nature of the TRCs remains obscure. Their unilateral distribution in this case supports the hypothesis that local factors are more important in their formation than systemic factors. In conjunction with other published work, this provides circumstantial evidence that irradiation may predispose to TRC deposition.
\end{abstract}

(F Clin Pathol 1994;47:362-364)

Tyrosine rich crystalloids (TRCs) have been described in various neoplasms of the major and minor salivary glands, with isolated examples in ceruminous and lacrimal gland tumours and in fibrous tissue in laryngectomy specimens. They may be seen in both histological sections and in cytological preparations but are not apparent macroscopically. They most commonly occur in pleomorphic adenomas, usually of the parotid gland. Personal experience and most studies suggest that TRCs may be demonstrated in approximately $1.5-5 \%$ of pleomorphic adenomas, although an incidence of $21 \%$ in tumours from African patients is reported in one study. ${ }^{1}$

Multiple, separate neoplasms of the sali-

Department of Pathology, The Medical School, University of Birmingham, Edgbaston, Birmingham B15 2TT

A T Warfield

L A Smallman

Correspondence to: Dr A T Warfield Department of Pathology, Staffordshire General Infirmary, Stafford

Infirmary, S
ST16 2PA.

Accepted for publication 14 October 1993 neck increases the risk of developing subsequent salivary neoplasms, and patients with radiation related salivary gland tumours experience a high rate of second tumours of salivary, thyroid, and parathyroid glands. ${ }^{3}$

To these authors' knowledge, simultaneous pleomorphic adenomas with TRCs confined to one neoplasm have never been described. Furthermore, any association between TRCs and irradiation of the head and neck has not previously been commented on.

\section{Case report}

A 41-year-old white man was referred for further investigation of painless, bilateral facial swelling. He was otherwise in good health but had a history of Hodgkin's lymphoma, which was diagnosed at the age of 12 years and had been treated with radiotherapy to cervicofacial fields. Over the following nine years relapse confirmed by biopsy occurred, followed by further radiation to the mediastinum and abdomen. Since that time he had been in clinical remission.

The right facial lump was in the region of the tail of the parotid gland and was first noticed three years previously. Initially, this was thought to represent another relapse of the Hodgkin's disease and radiotherapy was given empirically to the right side of the face after one year. Despite this, the lump continued to enlarge slowly over the next two years and, in addition, a similar left preauricular swelling became apparent. There was no other evidence to suggest relapse of Hodgkin's disease and therefore excision biopsy of both lesions was performed. The patient made an uneventful recovery.

\section{Pathological findings}

The right superficial parotidectomy specimen consisted of normal looking salivary gland with a $2.5 \mathrm{~cm}$ diameter, well circumscribed lesion towards one edge. This showed a solid, cream cut surface. The left lumpectomy specimen consisted of multiple cream tissue fragments, together measuring $1 \mathrm{~cm}$ in maximum dimension. All of the tissue was submitted for histological examination and multiple levels were cut through each block.

Microscopically, both specimens showed the typical heterogeneous appearances of pleomorphic adenomas. Myxoid stroma of low cellularity and more densely cellular areas were admixed in roughly equal proportions. The cellular areas showed a diverse pattern with solid sheets and anastomosing cords of epithelial cells, occasional clear cells, scattered groups of cells with a "plasmacytoid" 
appearance, and frequent duct like structures of varying size. The myxoid areas contained individual stellate and fusiform cells in a loose connective tissue background showing focal chondroid differentiation and patchy dystrophic calcification.

In addition, in the right side tumour there were localised collections of weakly refractile, non-birefringent crystal like structures (fig 1). These were present focally within the myxoid stroma in one of the four blocks. They were arranged either singly or in small aggregates and some appeared to have been fractured during tissue sectioning. Individual crystalloids were ovoid or round in outline and averaged approximately $30-45 \mu \mathrm{m}$ in diameter. They contained a sometimes indistinct, rounded core from which radiated multiple "floret like" or "rosette like" projections, giving a roughly symmetrical, lobulated contour. These structures were lightly eosinophilic, stained bright yellow-orange with Masson trichrome and Van Gieson preparations, and gave a deep purplish-red colour with the diazotisation coupling

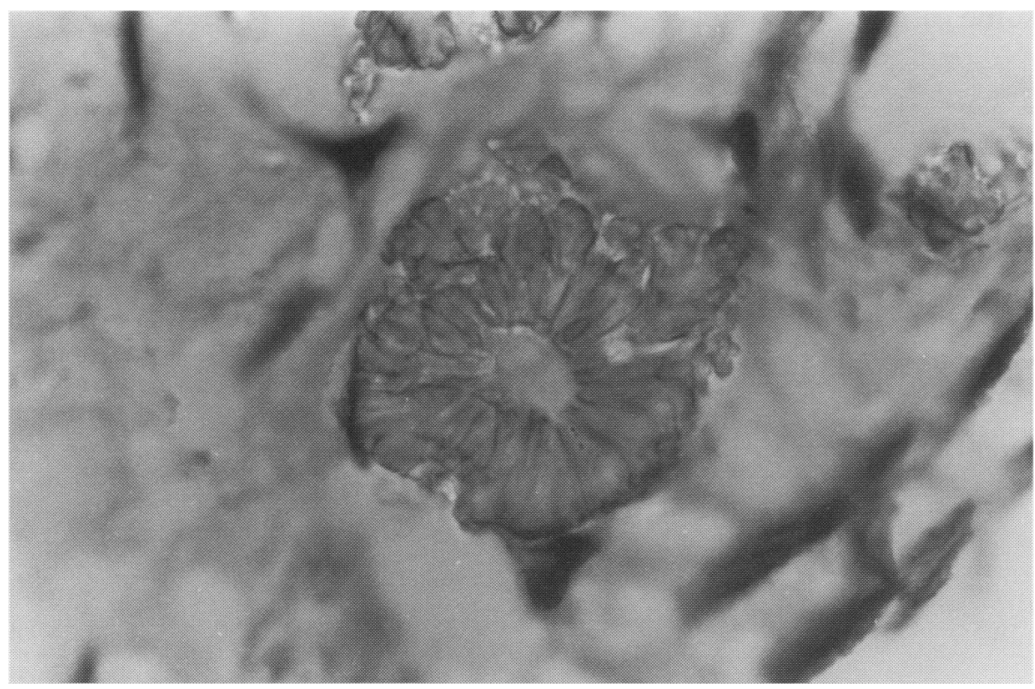

Figure 1 Individual, slightly refractile tyrosine rich crystalloid showing a typical "floret like" appearance (haematoxylin-Van Gieson).

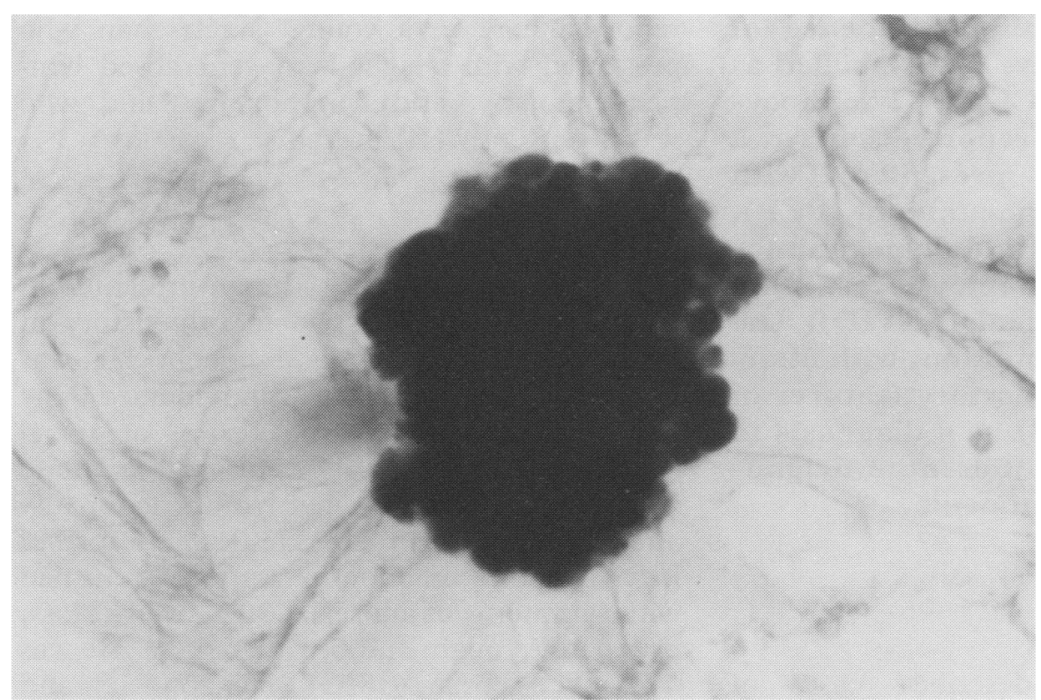

Figure 2 Individual tyrosine rich crystalloids showing a positive diazotisation coupling reaction. method for tyrosine (fig 2), consistent with TRCs. The surrounding salivary tissue showed mild fatty replacement but no other abnormality. The left side tumour showed similar histological features but without crystalloids. There was no evidence of Hodgkin's lymphoma.

\section{Discussion}

The pathogenesis and full importance of TRCs are largely unknown. They classically show positive results with the Millon reaction or diazotisation coupling method, indicating that tyrosine is present. Although originally thought to represent pure tyrosine crystals, a variety of techniques have subsequently demonstrated an amorphous polypeptide structure with a relative molecular weight in the region of $17000 .{ }^{4}$ This has a complex amino acid composition which, in addition to tyrosine, is also rich in arginine, tryptophan, and sulphydryl groups, and contains trace amounts of calcium, magnesium, and phosphorus. ${ }^{5}$ They show negative staining for collagen, although reticulin is present centrally and between individual "petals", and are refractile but not birefringent. ${ }^{6}$

TRCs should not be confused with morphologically similar collagenous crystalloids (stellate fibrillar formations), calcium oxalate crystals, or other poorly characterised intraductal crystalloids. Collagenous crystalloids are Millon/diazotisation negative, collagen positive, and weakly birefringent. Calcium oxalate crystals are Millon/diazotisation negative and collagen negative, but strongly birefringent. The diazotisation coupling method obviates the handling and disposal of toxic mercuric reagents used in the Millon reaction $^{7}$ and is therefore preferred in the authors' laboratory.

The previous therapeutic irradiation to the head and neck in this patient is a known risk factor for salivary gland neoplasia. It has been suggested that lower doses of radiation such as those used in certain diagnostic imaging techniques may also be tumourogenic to salivary tissues. ${ }^{8}$ The exposure effects are cumulative and the risk increases with dose. That the TRCs in this case were found only in the neoplasm given the highest radiation exposure may not be entirely coincidental. Several authors do mention previous radiotherapy ${ }^{9}$ or imaging procedures, such as full mouth dental radiography, ${ }^{10}$ in their TRC case reports, but make no comment as to any pathogenetic link. Although many of the patients described in the larger series would be expected to have had therapeutic or diagnostic irradiation, the cases are documented in insufficient detail to confirm any association.

Several tentative hypotheses to explain TRC formation have been proposed in the past. These include a defect of pigment metabolism, particularly as TRCs seem to be more common in pigmented races and salivary tyrosine secretion in albino people is reduced. Disordered protein synthesis 
by tumour cells or local changes in the concentration of mucopolysaccharides and inorganic ions in the matrix have also been suggested. Histological changes are usually not seen in salivary tissue following low dose radiation exposure. However, TRC deposition may be a more subtle indication of damage following higher doses of irradiation. Other local and possibly systemic factors might also be expected to contribute. The focal distribution of crystalloids in this case might suggest that local variables within an individual tumour may be important, in addition to differences between left and right sides. The precise details of any mechanism, however, remain obscure and purely speculative.

In conclusion, these findings provide circumstantial evidence to support the hypothesis that deposition of TRCs in salivary neoplasms is influenced more by local than by systemic factors. Furthermore, it is proposed that irradiation of the head and neck may be one predisposing factor in TRC formation, an observation that has not previously been made, and that crystalloid deposition may be a subtle reflection of radiation induced damage in some cases.
The authors thank Mr B Sims for his technical advice and assistance.

1 Thomas K, Hutt MSR. Tyrosine crystals in salivary gland tumours. 7 Clin Pathol 1981;34:1003-5.

2 Gnepp DR, Schroeder W, Heffner D. Synchronous tumours arising in a single major salivary gland. Cancer 1989;63:1219-24.

3 Auclair PL, Ellis GL, Gnepp DR, Wenig BM, Janney CG. Salivary gland neoplasms: general considerations. In: Ellis GL, Auclair PL, Gnepp DR, eds. Surgical pathology of the salivary glands. Philadelphia: WB Saunders, 1991 135-64.

4 Valente PT, Hoober JK, Phillips SJ. Tyrosine-rich crystalloids in pleomorphic adenoma: SEM findings and partial biochemical characterization. Ultrastruct Pathol 1988;12:613-20.

5 Humphrey PA, Ingram P, Tucker A. Crystalloids in salivary gland pleomorphic adenomas. Arch Pathol Lab Med vary gland pleom

6 Campbell WG, Priest RE, Weathers DR. Characterization of two types of crystalloids in pleomorphic adenomas of minor salivary glands. A light microscopic, electron microscopic and histochemical study. Am $\mathcal{F}$ Pathol 1985;118:194-202.

7 Pearse AGE. Proteins, peptides and amino acids. In (ed.), Vol. 2 Histochemistry, theoretical and applied. 4th edn. Edinburgh: Churchill Livingstone, 1985:441-537.

8 Preston-Martin S, Thomas DC White SC Cohen D. Prior exposure to medical and dental X-rays related to tumours of the parotid gland. $7 N C I$ 1990;80:943-9.

9 Bottles K, Ferrell LD, Miller TR. Tyrosine crystals in fine needle aspirates of a pleomorphic adenoma of the needle aspirates of a pleomorphic ad

10 Gould AR, Van Arsdall LR, Hinkle SJ, Harris WR Tyrosine-rich crystalloids in adenoid cystic carcinoma: histochemical and ultrastructural observations. $f$ Oral Pathol 1983;12:478-90.

\title{
CD4+ lymphocytopenia due to common variable immunodeficiency mimicking AIDS
}

\author{
R S Kaczmarski, A D B Webster, J Moxham, F Davison, S Sutherland, G J Mufti
}

\author{
Department of \\ Haematological \\ Medicine, King's \\ College School of \\ Medicine and \\ Dentistry, Denmark \\ Hill, London SE5 8RX \\ R S Kaczmarski \\ G J Mufti \\ Department of \\ Thoracic Medicine \\ J Moxham \\ Immunodeficiency \\ Research Group, \\ CRC, Northwick Park \\ Hospital, Harrow, \\ Middlesex \\ A D B Webster \\ Department of \\ Virology, Dulwich \\ Hospital, London \\ F Davison \\ S Sutherland \\ Correspondence to: \\ Dr G J Mufti

\begin{abstract}
There are an increasing number of published reports of patients with acquired immunodeficiency without evidence of HIV infection, who have been labelled as having "idiopathic CD4+ lymphocytopenia". The case is reported here of a young man who presented with Pneumocystis carinii pneumonia (PCP), CD4+ lymphopenia, and hypogammaglobulinaemia attributable to common variable immunodeficiency (CVID). The presentation of this condition, with many of the clinical and laboratory features of AIDS, highlights CVID as a diagnosis to be considered in the differential diagnosis of CD4 + lymphocytopenia.
\end{abstract}

(F Clin Pathol 1994;47:364-366)

Recent reports have described cases of acquired immunodeficiency without evidence of infection by HIV. However, other diag- noses can mimic the clinical characteristics of AIDS and must not be overlooked. A case of Pneumocystis carinii pneumonia (PCP) is reported here in a young, single man who presented with weight loss, generalised lymphadenopathy, and lymphopenia, and who was persistently HIV negative.

\section{Case report}

A previously fit 37 year old man presented in October 1989 with a two month history of pruritis, night sweats, and cough. He was unmarried, heterosexual, and denied any homosexual contact, drug abuse, or travel overseas. On examination he had bilateral axillary and supraclavicular lymphadenopathy and hepatosplenomegaly. He declined further investigation. Six months later he returned with symptoms of anorexia, $20 \mathrm{~kg}$ weight loss, productive cough, and breathlessness. He was cachectic, febrile $\left(38^{\circ} \mathrm{C}\right)$, tachypnoeic, and had bilateral inguinal lymphadenopathy. Chest examination showed bilateral crackles 